\title{
Solid Water-Insoluble Particles in Hailstones and Their Geophysical Significance
}

\author{
J. Rosinski \\ National Center for Aimospheric Research, Boulder, Colo. \\ (Manuscript receiverl 6 December 1965, in revised form 11 March 1966)
}

\section{ABSTRACT}

\begin{abstract}
By melting concentric layers of ice, the size distribution and concentration of solid water-insoluble particles accumulated in hailstones were determined for three hailstorms in Colorado. It was found that in approximately 50 per cent of the hailstones analyzed, particle concentration in ice increased with radial distance. Following an equation derived for this category of hailstones, a relationship is shown among liquid water content of a cloud, concentration of solid particles in cloud droplets, speed of the hailstone, and its residence time in the atmosphere. Spatial distribution of solid particles in hailstones was also determined by slicing hailstones and subsequently separating particles from the ice by sublimation under low pressure. It was found that some of the hailstones analyzed were oriented during their growth.
\end{abstract}

\section{Introduction}

Detailed study of the structure and composition of hailstones has increased our understanding of the processes of hail formation. Studies have been carried out, for example, on the density, liquid water content, shape and air bubble characteristics of various layers of individual hailstones. In the present paper an investigation is reported of the spatial distribution of solid water-insoluble particles within hailstones.

Let us consider briefly what is already known about hail formation (List, 1965). Hailstones are built up by accretion of cloud droplets, i.e., supercooled water droplets and ice crystals, and raindrops. The starting nucleus is a small hail particle originating from graupel. A small hail particle (up to $0.5 \mathrm{~cm}$ ) represents an interphase between graupel and hailstone. It is distinguished from graupel by the higher density and partially glazed surface, and from the hailstone by size. Craupel is a pellet of diameter up to $0.5 \mathrm{~cm}$ consisting of frozen cloud droplets united together. It originates in either an ice crystal or a frozen droplet. The crystallographic structure of a hailstone is layered, so that shells of small single crystals are often accompanied by layers of air bubbles and layers of palisade-like single crystals arranged radially and separated by clear ice regions.

The structure of air bubbles and their significance was discussed by List (1961), when he pointed out that similar conditions of ice growth produce ice having the same constant density, which means having on an average the same proportion of air distributed in the form of air bubbles in ice. In zones where air bubbles preserve the same character, growing conditions were similar. Where there is a discontinuity in a shell of bubbles, it can be safely assumed that the conditions of growth of an adjacent layer changed. These changes will govern the final shape of a hailstone. Shape, mass and volume determine the terminal velocity of a hailstone, its orientation during fall, and airflow trajectories around it. Therefore, they are of decisive importance in interpretation of icing conditions from individual ice shells confined by lines of air bubbles.

The first evidence of nonuniform distribution of aerosol particles present in hailstones was given by Vittori and Pesaresi (1964), who determined distribution of chlorides inside hailstones. They concluded that "wet" growth zones of the hailstones contain less salt than the "dry" growth ones.

It was thought that it might be possible to obtain additional information about the rate and mode of growth of a hailstone if, in addition to studying its structure, density, liquid water content, shape, etc., measurements were made of the spatial distribution of solid insoluble particles within a hailstone.

The results of preliminary experiments performed with hailstones collected during hailstorms in Colorado around Boulder (B-hailstones) and Fort Collins (CSUColorado State University hailstones) are described below.

\section{Experimental method}

Hailstones were collected on grass surfaces from different hailstorms, and transported to the laboratory in cold boxes. They were subsequently transferred into freezers for storage. In all cases, any liquid water present in a hailstone was frozen. The quantity of liquid water in Colorado hailstones is very small, if any (G. Goyer, private communication). Before melting a stone, visible pieces of grass or sand were removed from its surface. 


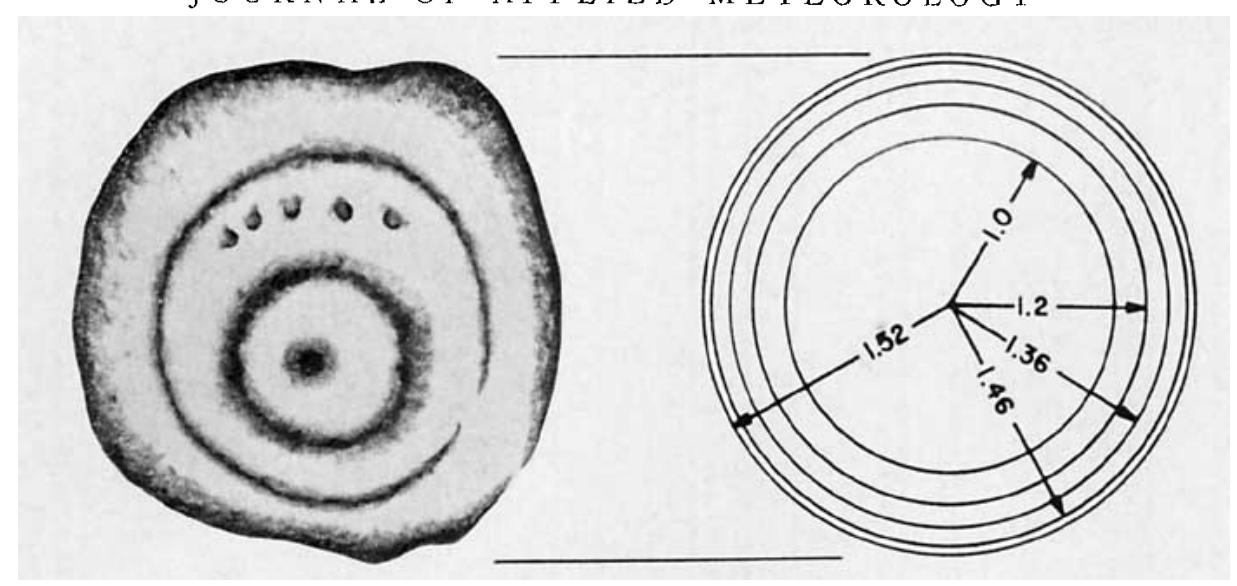

IFIG. 1. Ice shells (in $\mathrm{cm}$ ) melted off the hailstone (CSU-6.21.64 No. 4).

A hailstone was then dropped into a dry hemispherical Coulter-counter beaker heated to $30 \mathrm{C}$, and rotated rapidly by means of two glass rods until a thin layer of ice had been melted. The residual stone was lifted from that beaker and transferred into the next beaker, where another layer of ice was melted. This process was continued until the small core was melted in the last beaker. The concentration of different particle size classes was then determined for each layer of ice. No differences were found when the stones were melted and the melt-water was analyzed in the laboratory and in a particle-free box.

It has to be realized that part of the water from the melted ice can enter a hailstone and fill its air bubbles. To prevent or minimize loss of water into the interior of a hailstone during the process of melting off ice layers, hailstones were cooled to $-20 \mathrm{C}$ prior to melting. Thus, water entering the pores of a hailstone was refrozen and blocked the channels.

Different methods of melting were tried but did not quantitatively separate particles from the stone. Not all particles were removed from the surface of ice and transferred into water during melting of hailstones in warm air. Melting the hailstones in different liquids was unsatisfactory because it was not possible to determine the volume of a melted layer. Use of ultrasonic energy to remove layers of ice was unsatisfactory.

The quantity of ice melted (water) was determined by weighing Coulter beakers. A particle-free wetting agent, formaldehyde, and sodium chloride solution were then added to the water in beakers. A small quantity of formaldehyde was added to prevent the growth and multiplication of bacteria. The concentration and size distribution of solid insoluble particles was determined using the Coulter Particle Counter, Model B (Coulter Electronics, Inc., Chicago, Illinois). The Coulter counter determines the number and size of particles suspended in an electrically conductive liquid (sodium chloride solution), which is forced to flow through a small aperture. As a particle passes through the aperture, it changes the resistance between electrodes immersed on either side of it. This produces a voltage pulse of short duration of magnitude proportional to particle volume. The pulse height is then electronically scaled and counted. Size groups of particles were arbitrarily chosen to be: $5-13 \mu, 13-45 \mu, 45-100 \mu$ and larger than $100 \mu$ diameter. Accuracy of the count for the first group $(5-13 \mu)$ was \pm 5 per cent; for the remaining groups it was \pm 15 per cent. Upon completion of size and concentration analysis of particles up to $100 \mu$ diameter, the liquid was filtered through a membrane filter and particles of diameter larger than $100 \mu$ were counted under the microscope and the largest particle found in each layer was recorded.

In addition to this analysis, hailstones were sliced using the hot wire technique. Slices $1.5 \mathrm{~mm}$ thick were placed on a membrane filter and ice was sublimed off at $-20 \mathrm{C}$ at low pressure. Area density (number of particles per $\mathrm{cm}^{2}$ ) was then determined radially every 45. Particles were classified into four size classes: $d<5 \mu, 5 \leq d<10 \mu, 10 \leq d<5 \mu$, and $d \geq 25 \mu$ diameter.

To allow analysis of particle size distribution in a hailstone slice, the ice zones which follow air bubble distribution in a slice can be separated by a hot knife or by a surgical electric knife. Such a procedure may be the only method of following the visible mode of growth of a hailstone. When the surgical knife is used, the hailstone slice has to be placed on a metal block covered with a thin layer of particle-free sodium chloride solution or other conductive liquid. Conductive liquid should also be present on the surface of a slice.

\section{Experimental results and discussion}

The Coulter counter cannot distinguish between pollen, spores, clay, sand and other particles. To obtain some idea what living micro-organisms can be present in hailstones, a literature search was made. According to Gregory (1961), the organisms in precipitation water remain almost unstudied. The highest counts recorded are from hail. Even so, the numbers of micro-organisms found in hailstones collected in Canada (up to $10^{3}$ organisms per $\mathrm{ml}$ ), are far below particle concentrations found in hailstones in Colorado (up to $10^{6}$ particles 
per $\mathrm{ml}$ ). It can be assumed that collected material represents mainly vegetation debris (pollen, spores, etc.) and soil particles lifted by winds and removed by water drops in rain-out (incloud scavenging) and washout, and also scavenged by snow crystals and directly by hailstones. Therefore, counting and sizing were not affected by live bacteria which could multiply during the short time of sample preparation.

During the melting process it was noticed that the ice layers do not melt at the same rate. Whether or not melting follows density layers is unknown, so the amount of melted ice was chosen arbitrarily as the melting process proceeded. Spherical hailstones were used because the melting of outer layers was most easily controlled. A typical cross section of a hailstone (CSU-6.21.64 No. 4) is shown in Fig. 1. For the purpose of graphical presentation, thickness of concentric layers was calculated from the quantity of water obtained from melting the hailstone. It should be pointed out that outer layers correspond to the actual layers in a hailstone. The center of growth of a stone, though, is probably displaced in each case. At this time it is not possible to determine to what extent changes in concentration found around the center are affected by this displacement. Because of this uncertainty, emphasis was placed on the relationship between radial distance and particle concentration represented by a straight line (Fig. 2). This relationship was found in approximately 50 per cent of more than 100 analyzed hailstones. This type of hailstone will be the subject of the analysis below. Probably, different explanations could be proposed for the steady increase of the concentration of solid particles from a certain radial distance denoted here as $R_{\mathbf{0}}$. It is assumed that this type of growth takes place under the following conditions:

1) A hailstone grows by accretion of water drops during the final stage of its growth (from $R_{0}$ to $R$ ). Water drops scavenged by a growing hailstone are primarily cloud droplets consisting of supercooled water.

2) Cloud droplets contain particulate matter of concentration $N \mathrm{~cm}^{-3}$.

3) When a cloud droplet collides with and is captured by a hailstone, all solid particles are transferred from the droplet into the surface layer on a hailstone.

4) The number of collisions per unit time increases with the growing hailstone and is proportional to its cross section and velocity.

5) Part of the water is removed from the surface by means of evaporation or sublimation, leaving solid particles behind.

Another mechanism capable of displacing particles within a growing hailstone should be considered. Particles deposited on the surface of an ice-water interphase in a hailstone will be displaced from the position of their deposition by a growing ice front and will be under the influence of a temperature gradient (Hoekstra

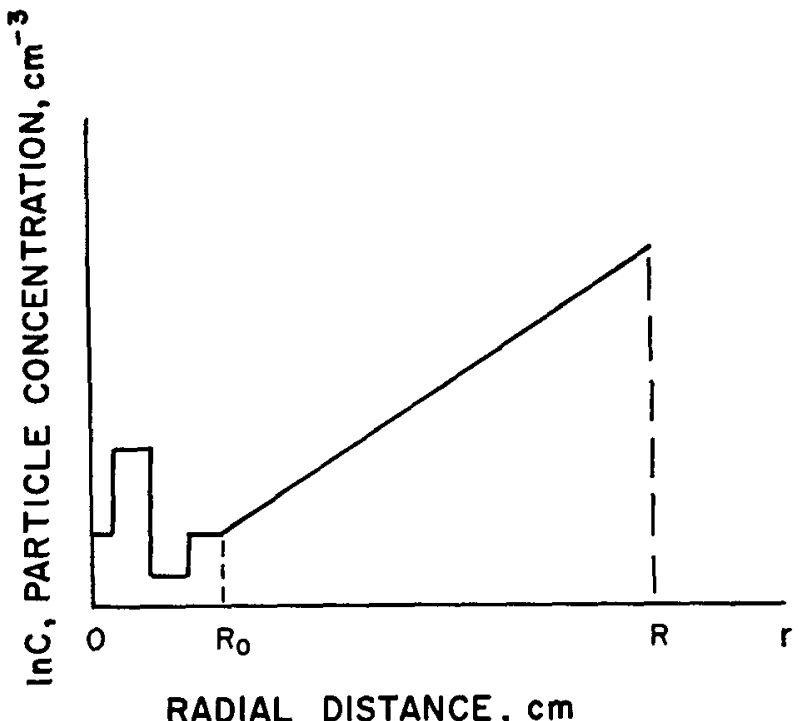

FIG. 2. Schematic drawing of the concentration of solid particles vs. radial distance found in typical hailstones.

and Miller, 1965; Uhlmann et al., 1964). This process, which cannot cause the uniform radial increase of particle concentration, can produce shells of particles corresponding to different stages of hailstone growth. In this case, the highest concentration should be found at the surface when all water present at that time had frozen. Presence of such layers was detected during microscopic examination of some hailstones.

Collision with a raindrop is probably very rare during this growth. But in such a case, it can be assumed that part of the water splashes away leaving a majority of particles on the surface of the hailstone. This is true only when fresh, unwettable soil particles are scavenged by a raindrop and are present on its surface. This process combined with evaporation should also produce a concentration gradient of solid particles primarily by mechanical removal of water, providing water can freeze on the surface of a hailstone. In this case the final growth should take place during the fall of the hailstone through rain. It is expected, however, that hailstones will melt under this condition. Growth of a hailstone by accretion of ice crystals must be accompanied by sublimation or melting and subsequent evaporation of water to account for distribution of particles, represented by a straight line, found in hailstones.

On the basis of this concept an equation was derived to calculate the residence time of a hailstone in the atmosphere during its final stages of growth.

\section{List of Symbols}

$r=$ radial distance, $\mathrm{cm}$

$R=$ radius of a hailstone, $\mathrm{cm}$

$W=$ volume of a hailstone, $\mathrm{cm}^{3}$

$C_{\text {water }}=$ concentration of particles in rain water, $\mathrm{cm}^{-3}$ 
$C_{\text {ice }}=$ concentration of particles in ice (hailstone) $\mathrm{cm}^{-3}$

$V=$ average speed of a hailstone, $\mathrm{cm} \mathrm{sec}^{-1}$

$v_{\mathrm{av}}=$ volume of an average cloud droplet, $\mathrm{cm}^{3}$

$\alpha=$ fraction

$A=$ concentration of cloud droplets, $\mathrm{cm}^{-3}$

$E=$ aerodynamic capture efficiency of water droplets by a hailstone, fraction

$N=$ average number of solid particles in one cloud droplet

$Q=$ liquid water content in clouds, $\mathrm{cm}^{3} \mathrm{~m}^{-3}$

$t=$ time, sec

$n=$ number of particles

$\beta, \gamma=$ constants

It was found experimentally that the concentration of solid particles in the hailstone increases from radial distance $r=R_{0}$ outward to the periphery of a hailstone $(r=R)$. It is expressed by an equation

$$
C_{\text {ice }}=\beta e^{\gamma r} \text {. }
$$

Values for constants $\beta$ and $\gamma$ calculated from graphs are given in Table 1.

With the assumptions given previously it can be reasoned that the rate of growth of a spherical hailstone is proportional to the cross section of a sphere of radius $r$ and of its speed of travel. Aerodynamic capture efficiency $E$ is not considered at this time. It should be noted, however, that in the case of a growing hailstone, $E$ is changing continuously with time.

In time $d t$, a hailstone will sweep all water particles $(E=1.0)$ from a cylinder of a base $\pi r^{2}$. The actual

TABLE 1. Values of $\beta$ and $\gamma$ in $C_{\text {iec }}=\beta e^{\gamma r}$

\begin{tabular}{|c|c|c|c|}
\hline \multirow{2}{*}{$\begin{array}{l}\text { Hailstone } \\
\text { number }\end{array}$} & \multirow{2}{*}{$\begin{array}{l}\text { Particle } \\
\text { size }(\mu)\end{array}$} & \multicolumn{2}{|c|}{ Constants } \\
\hline & & $\beta$ & $\gamma$ \\
\hline $\begin{array}{c}\text { CSU-6.21.64 } \\
1\end{array}$ & $5-13$ & $(-1) 1.3$ & 7.861 \\
\hline 2 & $5-13$ & (2) 2.3 & 4.439 \\
\hline 3 & $\begin{array}{c}5-13 \\
13-45 \\
45-100 \\
100-\end{array}$ & $\begin{array}{ll}(-3) & 3.4 \\
(-5) & 1.0 \\
(-4) & 2.0 \\
(-4) & 1.5\end{array}$ & $\begin{array}{l}13.743 \\
15.011 \\
10.012 \\
11.081\end{array}$ \\
\hline 4 & $\begin{array}{c}5-13 \\
13-45 \\
45-100 \\
100-\end{array}$ & $\begin{array}{rr}\text { (3) } & 1.7 \\
& 0.5 \\
(-2) & 2.0 \\
(-2) & 1.5\end{array}$ & $\begin{array}{l}3.903 \\
6.607 \\
7.238 \\
7.664\end{array}$ \\
\hline 5 & $\begin{array}{c}5-13 \\
13-45 \\
45-100\end{array}$ & $\begin{array}{rr}(-6) & 7.0 \\
(-9) & 1.0 \\
(-13) & 1.8\end{array}$ & $\begin{array}{l}11.521 \\
14.898 \\
16.629\end{array}$ \\
\hline $\begin{array}{c}\text { CSU-7.8.64 } \\
3\end{array}$ & $\begin{array}{c}5-13 \\
13-45 \\
45-100 \\
100-\end{array}$ & $\begin{array}{l}\text { (3) } 1.4 \\
\text { (1) } 3.7 \\
\text { (0) } 6.0 \\
\text { (1) } 2.8\end{array}$ & $\begin{array}{l}7.119 \\
6.695 \\
2.476 \\
4.504\end{array}$ \\
\hline $\begin{array}{c}\mathrm{B}-6.30 .65 \\
7\end{array}$ & $\begin{array}{r}5-13 \\
13-45\end{array}$ & $\begin{array}{l}\text { (4) } 7.7 \\
\text { (2) } 1.8\end{array}$ & $\begin{array}{l}2.135 \\
2.998\end{array}$ \\
\hline
\end{tabular}

The numbers in parentheses indicate the power of 10 by which tabulated values are to be multiplied.
Table 2. Particulate concentration of matter in hailstones (storm: CSU-6.21.64).

\begin{tabular}{|c|c|c|c|c|c|}
\hline \multirow{2}{*}{$\begin{array}{c}\text { Hail- } \\
\text { stone } \\
\text { number }\end{array}$} & \multirow{2}{*}{$\begin{array}{c}\text { Radial } \\
\text { distance } \\
(\mathrm{cm})\end{array}$} & \multicolumn{4}{|c|}{$\begin{array}{c}\text { Concentration }\left(\mathrm{cm}^{-3}\right) \text { for particle } \\
\text { diameter range }(\mu)\end{array}$} \\
\hline & & $5-13$ & $13-45$ & $45-100$ & 100 \\
\hline 1 & $\begin{array}{r}1.78-1.92 \\
1.61-1.78 \\
1.46-1.61 \\
1.11-1.46 \\
0-1.11 \\
0-1.92\end{array}$ & $\begin{array}{ll}\text { (5) } & 2.92 \\
\text { (5) } & 1.03 \\
\text { (4) } & 7.09 \\
\text { (4) } & 8.04 \\
\text { (4) } & 4.72 \\
\text { (5) } & 1.19\end{array}$ & $\begin{array}{ll}\text { (3) } & 8.71 \\
\text { (3) } & 1.49 \\
\text { (3) } & 1.54 \\
\text { (3) } & 2.27 \\
\text { (2) } & 7.26 \\
\text { (3) } & 2.95\end{array}$ & $\begin{array}{ll}\text { (2) } & 4.62 \\
\text { (2) } & 4.51 \\
\text { (2) } & 2.06 \\
\text { (2) } & 3.60 \\
\text { (2) } & 1.30 \\
\text { (2) } & 3.22\end{array}$ & $\begin{array}{ll}\text { (2) } & 4.08 \\
(2) & 1.61 \\
(2) & 1.25 \\
\text { (1) } & 7.49 \\
& 7.2 \\
\text { (2) } & 1.55\end{array}$ \\
\hline 2 & $\begin{array}{r}1.46-1.63 \\
1.21-1.46 \\
0.87-1.21 \\
0-0.87 \\
0-1.63\end{array}$ & $\begin{array}{l}\text { (5) } 2.19 \\
\text { (4) } 8.22 \\
\text { (4) } 2.31 \\
\text { (3) } 9.86 \\
\text { (4) } 8.35\end{array}$ & $\begin{array}{ll}\text { (3) } & 3.84 \\
\text { (3) } & 2.02 \\
\text { (2) } & 4.09 \\
\text { (2) } & 4.58 \\
\text { (3) } & 1.68\end{array}$ & $\begin{array}{l}\text { (2) } 2.65 \\
\text { (2) } 1.73 \\
\text { (1) } 3.89 \\
\text { (1) } 2.20 \\
\text { (2) } 1.25\end{array}$ & $\begin{array}{ll}\text { (2) } & 3.85 \\
\text { (2) } & 3.32 \\
\text { (1) } & 1.36 \\
\text { (1) } & 1.64 \\
\text { (2) } & 1.87\end{array}$ \\
\hline 3 & $\begin{array}{r}1.31-1.43 \\
1.08-1.31 \\
0.84-1.08 \\
0-0.84 \\
0-1.43\end{array}$ & $\begin{array}{l}\text { (5) } 4.67 \\
\text { (4) } 4.83 \\
\text { (5) } 2.37 \\
\text { (5) } 2.21 \\
\text { (5) } 2.43\end{array}$ & $\begin{array}{ll}\text { (3) } & 7.50 \\
\text { (2) } & 5.81 \\
\text { (2) } & 3.90 \\
\text { (1) } & 4.0 \\
\text { (3) } & 2.13\end{array}$ & $\begin{array}{ll}\text { (2) } & 1.70 \\
\text { (1) } & 2.96 \\
\text { (1) } & 3.32 \\
\text { (1) } & 2.0 \\
\text { (1) } & 6.32\end{array}$ & $\begin{array}{ll}\text { (2) } & 5.45 \\
\text { (1) } 8.71 \\
\text { (1) } 4.74 \\
\text { (1) } 2.88 \\
\text { (2) } 1.77\end{array}$ \\
\hline 4 & $\begin{array}{r}1.47-1.53 \\
1.36-1.47 \\
1.22-1.36 \\
1.02-1.22 \\
0-1.02 \\
0-1.53\end{array}$ & $\begin{array}{l}\text { (5) } 5.54 \\
\text { (5) } 3.86 \\
\text { (5) } 2.49 \\
\text { (5) } 1.33 \\
\text { (4) } 3.55 \\
\text { (5) } 2.72\end{array}$ & $\begin{array}{ll}\text { (3) } & 9.96 \\
\text { (3) } & 6.95 \\
\text { (3) } & 2.71 \\
\text { (2) } & 6.89 \\
\text { (2) } & 1.41 \\
\text { (3) } & 4.09\end{array}$ & $\begin{array}{ll}\text { (2) } & 6.61 \\
\text { (2) } & 6.61 \\
\text { (2) } & 2.07 \\
\text { (1) } & 4.81 \\
\text { (1) } & 2.52 \\
\text { (2) } & 3.20\end{array}$ & $\begin{array}{ll}\text { (3) } & 1.26 \\
\text { (2) } & 7.45 \\
\text { (2) } & 2.16 \\
\text { (1) } & 5.35 \\
\text { (1) } & 2.31 \\
\text { (2) } & 4.60\end{array}$ \\
\hline 5 & $\begin{array}{r}2.12-2.17 \\
2.04-2.12 \\
1.94-2.04 \\
1.81-1.94 \\
1.68-1.81 \\
1.53-1.68 \\
1.35-1.53 \\
1.12-1.35 \\
0.81-1.12 \\
0-0.81 \\
0-2.17\end{array}$ & $\begin{array}{ll}\text { (5) } & 5.75 \\
\text { (5) } & 2.77 \\
\text { (4) } & 7.24 \\
\text { (4) } & 1.38 \\
\text { (4) } & 3.29 \\
\text { (3) } & 5.60 \\
\text { (4) } & 4.16 \\
\text { (4) } & 3.19 \\
\text { (5) } & 4.82 \\
\text { (5) } & 2.15 \\
\text { (5) } & 1.75\end{array}$ & $\begin{array}{ll}\text { (4) } & 1.32 \\
\text { (3) } & 3.72 \\
\text { (3) } & 1.38 \\
\text { (2) } & 4.61 \\
\text { (1) } & 8.90 \\
\text { (1) } & 4.67 \\
\text { (2) } & 1.45 \\
\text { (1) } & 7.32 \\
\text { (2) } & 1.42 \\
\text { (2) } & 2.37 \\
\text { (3) } & 1.95\end{array}$ & $\begin{array}{ll}\text { (2) } & 6.37 \\
\text { (2) } & 1.80 \\
\text { (1) } & 2.9 \\
\text { (1) } & 2.01 \\
\text { (1) } & 3.24 \\
\text { (1) } & 1.63 \\
\text { (1) } & 1.82 \\
\text { (1) } & 1.37 \\
\text { (1) } & 1.37 \\
\text { (1) } & 4.10 \\
\text { (2) } & 1.00\end{array}$ & $\begin{array}{ll}\text { (2) } & 2.19 \\
\text { (1) } & 7.01 \\
\text { (1) } & 4.17 \\
\text { (1) } & 2.80 \\
\text { (1) } & 1.78 \\
\text { (1) } & 2.54 \\
\text { (1) } & 3.65 \\
\text { (1) } & 2.61 \\
\text { (1) } & 2.60 \\
\text { (1) } & 2.47 \\
\text { (1) } & 5.15\end{array}$ \\
\hline
\end{tabular}

The numbers in parentheses indicate the power of 10 by which tabulated values are to be multiplied.

volume of all swept water particles is

$$
E v_{\mathrm{av}} A \pi r^{2} V d t
$$

where the height of a cylinder $d \mathrm{~L}$ is $V d t$. Part $\alpha$ of this volume contributes to the growth of a hailstone (part $[1-\alpha]$ being evaporated or removed by mechanical forces). Therefore,

where

$$
d W=E \alpha v_{\mathrm{av}} A \pi r^{2} V d t
$$

$$
\text { and }
$$

$$
W=\frac{4}{3} \pi r^{3},
$$$$
\frac{4}{E A \alpha v_{\mathrm{av}}} d r=V d l \text {. }
$$

But

$$
C_{\text {water }}=\frac{N}{v_{\mathrm{ut}}}
$$

and

$$
C_{\mathrm{ice}}=\frac{N}{\alpha v_{\mathrm{av}}}
$$

therefore,

$$
\frac{4 C_{\mathrm{ice}}}{E A N} d r=V d t
$$


Substituting $C_{\text {ice }}$ from $\mathrm{Eq}$. (1) into $\mathrm{Eq} .(6)$,

$$
\frac{4 \beta e^{\gamma r}}{E A I^{r}} d t=V d t
$$

and integrating between limits

$$
\frac{4 \beta}{E A N^{\top}} \int_{r=R_{0}}^{r=R} e^{\gamma r} d r=V \int_{t=0}^{t=t} d t
$$

we have the relationship ( $\boldsymbol{V}$ is the average value of speed and is constant for each hailstone; it can be an average velocity acquired during the residence time in an updraft or falling average terminal velocity):

$$
\frac{4 \beta\left(e^{\gamma R}-e^{\gamma R_{0}}\right)}{E A_{\perp} \gamma}=V t
$$

The liquid water content of a cloud is

$$
Q=A r_{\mathrm{av}}
$$

Substituting Eys. (12) and (6) into (11), we have

$$
\frac{4 \beta}{E \gamma Q C_{\text {water }}}\left(e^{\gamma R}-e^{\gamma R 0}\right)=V t
$$

The results from the analysis of three hailstorms are given in Tables 2, 3 and 4 and Figs. 3, 4 and 5. The largest particle found in each layer of ice is given in Table 5 and average values are shown in Fig. 6. Hailstone C.SL-6.21.64 No. 4 (Fig. 3) and particle size range $5 \sim 13 \mu$ will be used as an example. Eq. (13) gives the following information on time of growth of a hailstone

\begin{tabular}{|c|c|c|c|c|c|}
\hline \multirow{2}{*}{$\begin{array}{c}\text { Hail- } \\
\text { stone } \\
\text { number }\end{array}$} & \multirow{2}{*}{$\begin{array}{c}\text { Radial } \\
\text { distance } \\
(\mathrm{cm})\end{array}$} & \multicolumn{4}{|c|}{$\begin{array}{c}\text { Concentration }\left(\mathrm{cm}^{-3}\right) \text { for particle } \\
\text { diameter range }(\mu)\end{array}$} \\
\hline & & $5-13$ & $13-45$ & $45-100$ & $100-$ \\
\hline \multirow[t]{5}{*}{1} & $1.11-1.23$ & (5) 3.09 & (3) 8.44 & (2) 9.90 & (2) 3.43 \\
\hline & $0.87-1.11$ & (5) 2.46 & (3) 3.79 & (2) 1.77 & (1) 5.78 \\
\hline & $0.58-0.87$ & (5) 2.77 & (3) 8.36 & (1) 4.16 & (1) 2.5 \\
\hline & $0-0.58$ & (6) 1.11 & (3) 1.36 & (2) 1.58 & (1) 4.13 \\
\hline & $0-1.23$ & (5) 4.86 & (3) 5.49 & (2) 3.42 & (2) 1.17 \\
\hline \multirow[t]{5}{*}{2} & $1.08-1.16$ & (5) 5.11 & (3) 1.10 & (2) 7.53 & (2) 2.20 \\
\hline & $0.87-1.08$ & (5) 5.44 & (3) 2.83 & (2) 2.03 & (1) 7.08 \\
\hline & $0.65-0.87$ & (5) 7.30 & (3) 3.04 & (1) 9.35 & (1) 6.53 \\
\hline & $0-0.65$ & (5) 4.72 & (3) 1.00 & (1) 2.94 & (1) 7.50 \\
\hline & $0-1.16$ & (5) 5.64 & (3) 1.99 & (2) 2.70 & (2) 1.0 \\
\hline \multirow[t]{5}{*}{3} & $0.70-0.82$ & (5) 2.90 & (3) 5.94 & (2) 4.80 & (2) 8.93 \\
\hline & $0.58-0.70$ & (5) 1.30 & (3) 2.87 & (2) 2.40 & \\
\hline & $0.36-0.58$ & (4) 5.53 & (2) 8.30 & (1) 2.77 & (2) 2 . \\
\hline & $0-0.36$ & (4) 6.29 & (2) 6.80 & 0 & (2) 2.59 \\
\hline & $0-0.82$ & (5) 1.35 & (3) 2.58 & (2) 1.87 & (2) 4.75 \\
\hline
\end{tabular}
from $R_{0}$ to $R$. Particle concentration in liquid water in a cloud or ice crystals which originate the hailstone

TABLe 3. Particulate concentration of matter in hailstones (storm: CSU-7.8.64).

\begin{tabular}{|c|c|c|c|c|c|}
\hline \multirow{2}{*}{$\begin{array}{l}\text { Hail- } \\
\text { stone } \\
\text { number }\end{array}$} & \multirow{2}{*}{$\begin{array}{l}\text { Radial } \\
\text { distance } \\
(\mathrm{cm})\end{array}$} & \multicolumn{4}{|c|}{$\begin{array}{c}\text { Concentration }\left(\mathrm{cm}^{-3}\right) \text { for particle } \\
\text { diameter range }(\mu)\end{array}$} \\
\hline & & $5-13$ & $13-45$ & $45-100$ & $100-$ \\
\hline 1 & $\begin{array}{r}0.94-0.96 \\
0.88-0.94 \\
0.76-0.88 \\
0.61-0.76 \\
0.40-0.61 \\
0-0.40 \\
0-0.96\end{array}$ & $\begin{array}{ll}\text { (4) } & 3.42 \\
\text { (4) } & 1.64 \\
\text { (3) } & 9.71 \\
\text { (4) } & 1.40 \\
\text { (3) } & 6.77 \\
\text { (5) } & 1.08 \\
\text { (4) } & 3.15\end{array}$ & $\begin{array}{ll}\text { (2) } & 5.49 \\
\text { (2) } & 6.89 \\
\text { (2) } & 3.12 \\
\text { (2) } & 1.60 \\
0 \\
0 \\
\text { (2) } 2.85\end{array}$ & $\begin{array}{cl} & 0 \\
\text { (1) } & 1.32 \\
\text { (1) } & 5.20 \\
\text { (1) } & 1.00 \\
\text { (1) } & 7.25 \\
& 0 \\
\text { (1) } & 2.46\end{array}$ & $\begin{array}{ll}\text { (2) } & 1.54 \\
\text { (1) } & 9.17 \\
\text { (1) } & 5.45 \\
\text { (1) } 5.03 \\
\text { (2) } 1.06 \\
\text { (2) } 2.17 \\
\text { (2) } 1.12\end{array}$ \\
\hline 2 & $\begin{array}{r}1.00-1.05 \\
0.95-1.00 \\
0.85-0.95 \\
0.76-0.85 \\
0.56-0.76 \\
0-0.56 \\
0-1.05\end{array}$ & $\begin{array}{ll}\text { (4) } & 2.46 \\
\text { (4) } & 2.60 \\
\text { (4) } & 2.08 \\
\text { (4) } & 1.07 \\
\text { (4) } & 2.04 \\
\text { (3) } & 7.60 \\
\text { (4) } & 1.84\end{array}$ & $\begin{array}{ll}\text { (3) } & 1.96 \\
\text { (2) } & 9.88 \\
\text { (3) } & 1.79 \\
\text { (3) } & 1.11 \\
\text { (2) } & 9.89 \\
& 0 \\
\text { (3) } & 1.14\end{array}$ & $\begin{array}{ll}\text { (2) } & 2.26 \\
\text { (2) } & 1.82 \\
\text { (2) } & 1.10 \\
0 \\
0 \\
0 \\
\\
\text { (1) } 2.00\end{array}$ & $\begin{array}{ll}\text { (2) } & 1.23 \\
\text { (2) } & 1.01 \\
\text { (2) } & 1.20 \\
\text { (2) } & 1.29 \\
\text { (2) } & 1.10 \\
\text { (2) } & 1.47 \\
\text { (2) } & 1.22\end{array}$ \\
\hline 3 & $\begin{array}{r}0.47-0.70 \\
0-0.47 \\
0-0.70\end{array}$ & $\begin{array}{l}\text { (5) } 3.22 \\
\text { (5) } 5.85 \\
\text { (5) } 4.54\end{array}$ & $\begin{array}{ll}\text { (3) } & 2.38 \\
\text { (3) } & 1.63 \\
\text { (3) } & 2.00\end{array}$ & $\begin{array}{ll}\text { (2) } & 1.68 \\
\text { (2) } & 1.13 \\
\text { (2) } & 1.40\end{array}$ & $\begin{array}{l}\text { (1) } 6.06 \\
\text { (1) } 7.95 \\
\text { (1) } 7.00\end{array}$ \\
\hline 4 & $\begin{array}{r}0.57-0.71 \\
0-0.57 \\
0.0 .71\end{array}$ & $\begin{array}{l}\text { (5) } 2.38 \\
\text { (5) } 3.87 \\
\text { (5) } 3.12\end{array}$ & $\begin{array}{ll}\text { (3) } & 3.00 \\
\text { (2) } & 8.33 \\
\text { (3) } & 1.92\end{array}$ & $\begin{array}{ll}\text { (2) } & 1.64 \\
\text { (1) } & 7.81 \\
\text { (2) } 1.21\end{array}$ & $\begin{array}{l}\text { (2) } 2.66 \\
\text { (2) } 1.38 \\
\text { (2) } 2.02\end{array}$ \\
\hline 5 & $\begin{array}{r}0.71-0.81 \\
0.54-0.71 \\
0-0.54 \\
0-0.81\end{array}$ & $\begin{array}{ll}\text { (5) } & 6.24 \\
\text { (5) } & 2.82 \\
\text { (5) } & 3.26 \\
\text { (5) } & 4.11\end{array}$ & $\begin{array}{ll}\text { (4) } & 1.26 \\
\text { (3) } & 5.54 \\
\text { (3) } & 3.62 \\
\text { (3) } & 7.25\end{array}$ & $\begin{array}{l}\text { (2) } 3.68 \\
\text { (2) } 2.57 \\
\text { (2) } 3.88 \\
\text { (2) } 3.38\end{array}$ & $\begin{array}{l}\text { (1) } 6.2 \\
\text { (1) } 6.30 \\
\text { (1) } 9.9 \\
\text { (1) } 7.4\end{array}$ \\
\hline 6 & $\begin{array}{r}0.85-0.96 \\
0.73-0.85 \\
0.50-0.73 \\
0-0.50 \\
0-0.96\end{array}$ & $\begin{array}{ll}\text { (5) } & 1.72 \\
\text { (4) } & 9.56 \\
\text { (4) } & 8.25 \\
\text { (5) } & 1.49 \\
\text { (5) } & 1.25\end{array}$ & $\begin{array}{l}\text { (3) } 8.80 \\
\text { (2) } 8.94 \\
\text { (3) } 3.10 \\
\text { (3) } 4.58 \\
\text { (3) } 4.34\end{array}$ & $\begin{array}{l}\text { (2) } 4.83 \\
\text { (2) } 1.07 \\
\text { (1) } 8.80 \\
\text { (2) } 2.12 \\
\text { (2) } 2.22\end{array}$ & $\begin{array}{l}\text { (2) } 1.12 \\
\text { (1) } 5.40 \\
\text { (1) } 3.15 \\
\text { (1) } 9.19 \\
\text { (1) } 7.22\end{array}$ \\
\hline 7 & $\begin{array}{r}0.96-1.02 \\
0.83-0.96 \\
0.67-0.83 \\
0-0.67 \\
0-1.02\end{array}$ & $\begin{array}{ll}\text { (5) } & 6.23 \\
\text { (5) } & 4.78 \\
\text { (5) } & 4.25 \\
\text { (5) } & 3.51 \\
\text { (5) } & 4.69\end{array}$ & $\begin{array}{ll}\text { (3) } & 3.44 \\
\text { (3) } & 2.86 \\
\text { (3) } & 1.86 \\
\text { (3) } & 3.67 \\
\text { (3) } & 2.96\end{array}$ & $\begin{array}{ll}\text { (2) } & 1.36 \\
\text { (2) } & 2.07 \\
\text { (2) } & 1.52 \\
\text { (2) } & 2.55 \\
\text { (2) } & 1.88\end{array}$ & $\begin{array}{l}\text { (2) } 1.11 \\
\text { (1) } 5.68 \\
\text { (1) } 5.47 \\
\text { (1) } 8.0 \\
\text { (1) } 7.5 .\end{array}$ \\
\hline
\end{tabular}

The numbers in parentheses indicate the power of 10 by which tabulated values are to be multiplied.
TABLE 4. Particulate concentration of matter in hailstones (storm: B-6.30.65)

The numbers in parentheses indicate the power of 10 by which tabulated values are to be multiplied.

was found to have the values

$$
\begin{aligned}
C_{\text {water }} & =3.5 \times 10^{4} \text { particles } \mathrm{cm}^{-3}, \\
C_{\text {ice }} & =3.5 \times 10^{4} \text { to } 5.5 \times 10^{5} \text { particles } \mathrm{cm}^{-3},
\end{aligned}
$$

where $3.5 \times 10^{4}$ corresponds to concentration at the very beginning of the process of solid-particle concentration buildup and $5.5 \times 10^{5}$ to the outer shell of the hailstone.

From Eq. (5)

$$
\alpha=\frac{C_{\text {water }}}{C_{\text {ice }}} \text { and its value is between } 0.064 \text { and } 1.00 \text {. }
$$

This suggests that 6.4 per cent of water deposited stayed on the surface and contributed to the very final stages of hailstone growth and 93.6 per cent was evaporated or removed by some other processes. In addition to this, the rate of evaporation was changing continuously during the growth. Constants determined for this stone were: $\beta=1700$ and $\gamma=3.903$ while the hailstone grew 


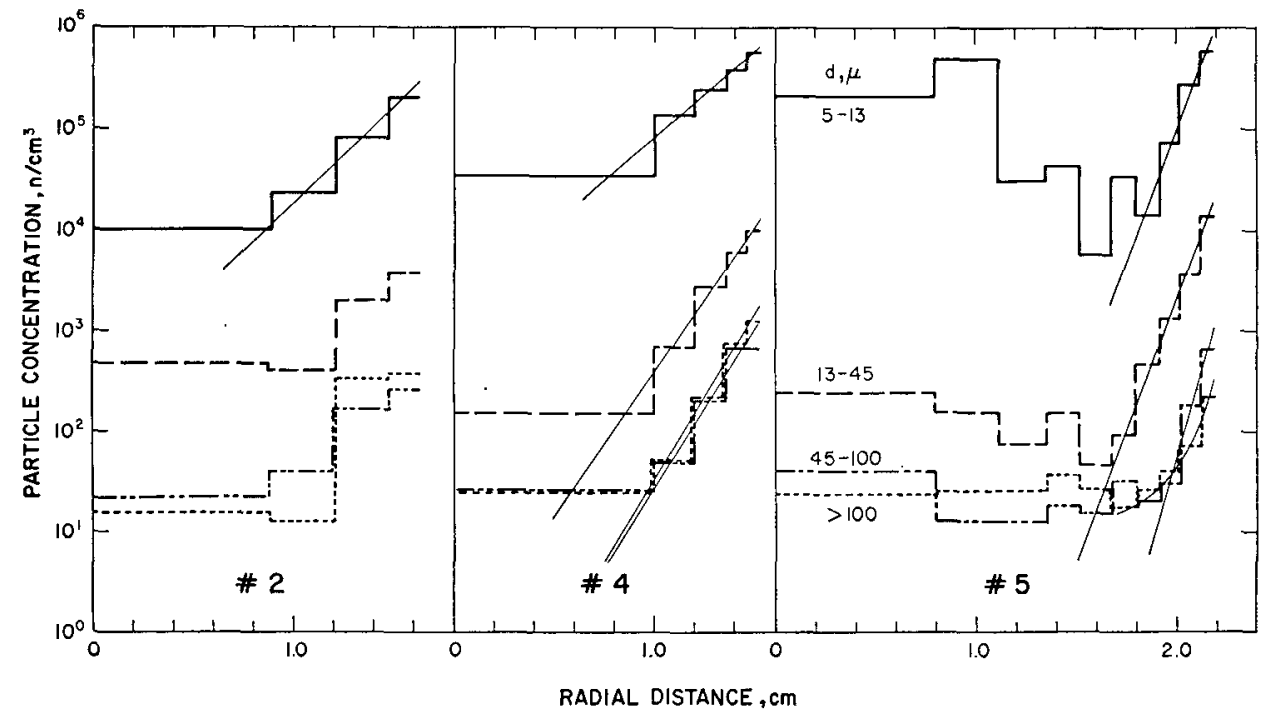

FıG. 3. Typical particle concentration of diameter vs. radial distance for hailstones from storm (CSU-6.21.64).

from $R_{0}=1.0 \mathrm{~cm}$ to $R=1.46 \mathrm{~cm}$. Substituting these values into Eq. (13), we obtain

and

$$
\frac{4 \times 1700}{E Q C_{\text {water }} 3.903}\left(e^{3.903 \times 1.46}-e^{3.903 \times 1.00}\right)=V l,
$$

$$
\frac{4.36 \times 10^{5}}{E Q C_{\text {water }}}=V t .
$$

One might think that the number of particles in cloud droplets can be estimated from the concentration of particles in rain water. 'This may be completely misleading but no measurement of concentration in cloud droplets was made and, therefore, the values of concentration found inside stones are used in this calculation. Liquid water contents in hail clouds are reported to be up to $30 \mathrm{gm} \mathrm{m}^{-3}$ (Battan, 1965). Assuming $E=1: 0$,

$$
Q=10 \mathrm{gm} \mathrm{m}^{-3}=10^{-5} \mathrm{~cm}^{3} \mathrm{~cm}^{-3},
$$

and using the experimentally found value

$$
C_{\text {water }}=3.5 \times 10^{4} \text { particles } \mathrm{cm}^{-3} \text {, }
$$

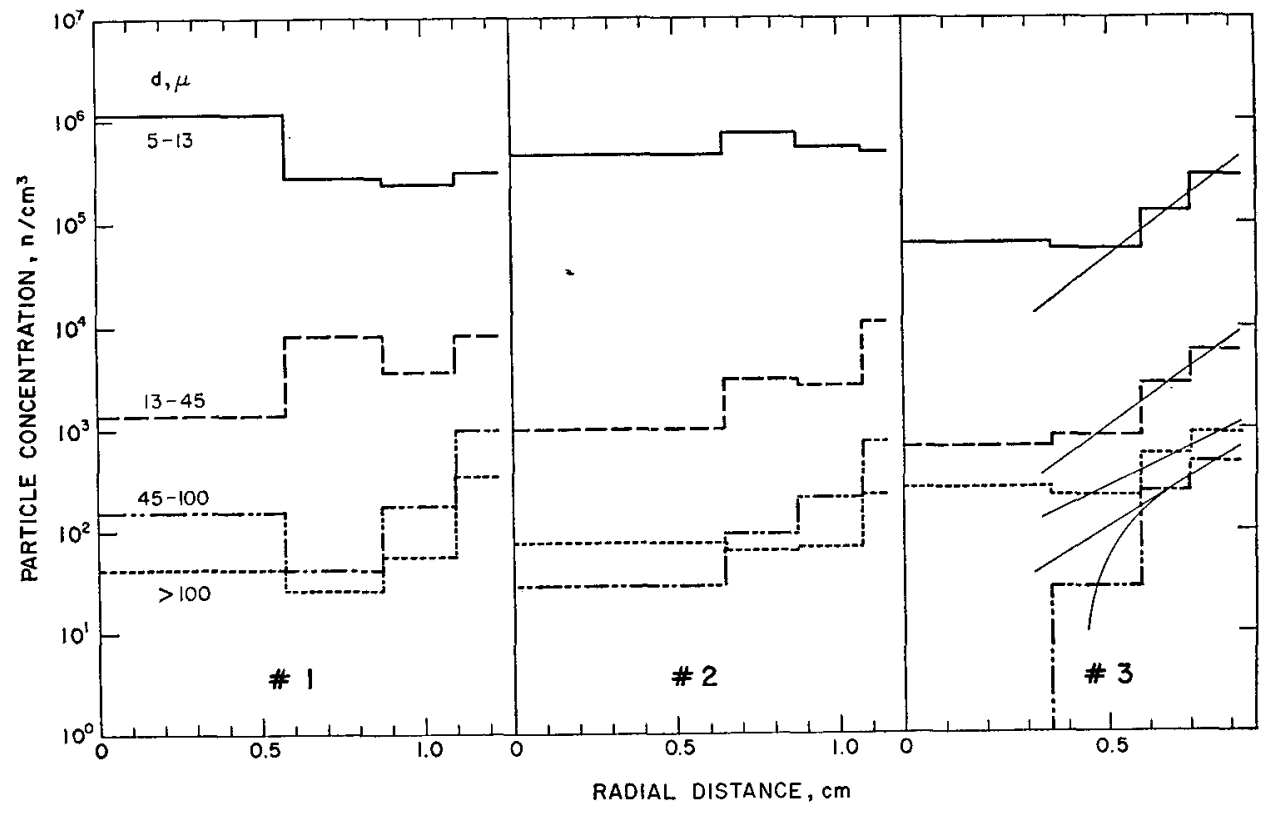

Fig. 4. Typical particle concentration vs. radial distance for hailstones from storm (CSU--7.8.64). 


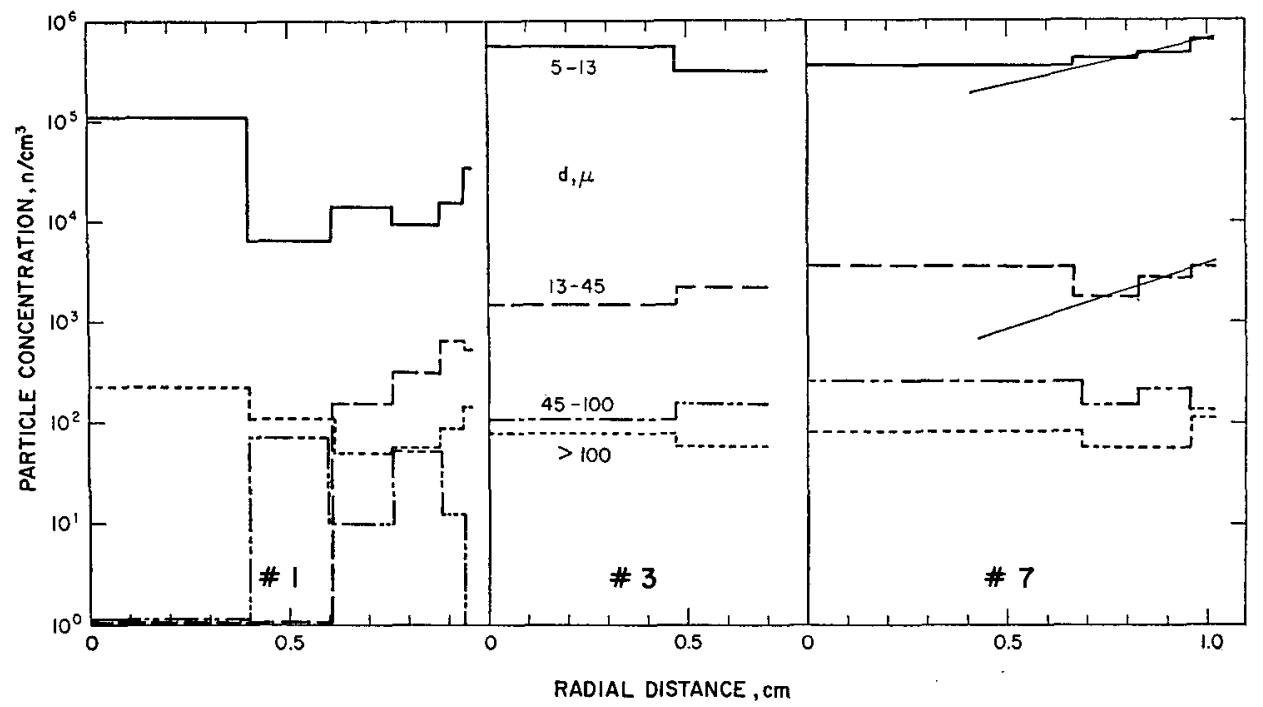

FIG. 5. Typical particle concentration vs. radial distance for hailstones from storm $(B-6.30 .65)$.

we have

$$
V t=\frac{4.36 \times 10^{5}}{10^{-5} \times 3.5 \times 10^{4}}=1.2 \times 10^{6} \mathrm{~cm} .
$$

This indicates that the growing hailstone travelled for a distance of $12 \mathrm{~km}$. Since the reported updraft and hailstone speeds are between 10 and $20 \mathrm{~m} \mathrm{sec}^{-1}$, the residence time was therefore between 10 and $20 \mathrm{~min}$. Correction for efficiency of capture of water droplets by a growing hailstone will increase $V t$ by a factor; for example, if the average value for $E$ is $0.8, V t=1.2 \times 10^{6} /$ $0.8=1.5 \times 10^{6} \mathrm{~cm}$ and the residence time is between 12 and $25 \mathrm{~min}$. Hailstone B-6.30.65 No. 7, which has the slowest buildup of solid particles in the $5-13 \mu$ diameter range of all hailstones analyzed, can be used as another example. The radial growth of the concentration of $5-13 \mu$ diameter size range followed the equation

$$
C_{\text {ice }}=7.7 \times 10^{4} e^{2.135 r} \text {. }
$$

The concentration of particles in cloud droplets was found to be $3.51 \times 10^{5}$ particles $\mathrm{cm}^{-3}$. For $Q=10 \mathrm{gm}$ $\mathrm{m}^{-3}$ and $10 \leq V \leq 20 \mathrm{~m} \mathrm{sec}-1$, the residence time is between 3 and $7 \mathrm{~min}$. The rate growth and the final size of a hailstone, as Eq. (13) predicts, depend strongly upon liquid water content of the cloud and the vertical gradient of air updraft velocities or terminal velocities of falling hailstones. The total time of growth of a hailstone should probably be more than twice the time of the growth of the outer layer from $R_{0}$ to $R$.

In the two cases calculated above, the liquid water content $Q$ was assumed as $10 \mathrm{gm} \mathrm{m}^{-3}$. This assumption cannot be true for both hailstorms analyzed. Capture of solid particles by a growing hailstone was assumed to be proportional to its cross section. In this assumption, efficiency of actual aerodynamic capture of droplets was not considered. The number of cloud droplets captured should actually be smaller. On the other hand, because the growth is taking place in a highly turbulent medium, the number may be compensated for by the capture of additional particles or even surpass the number swept by a cross section of a hailstone. It would be superfluous to consider efficiency of aerodynamic

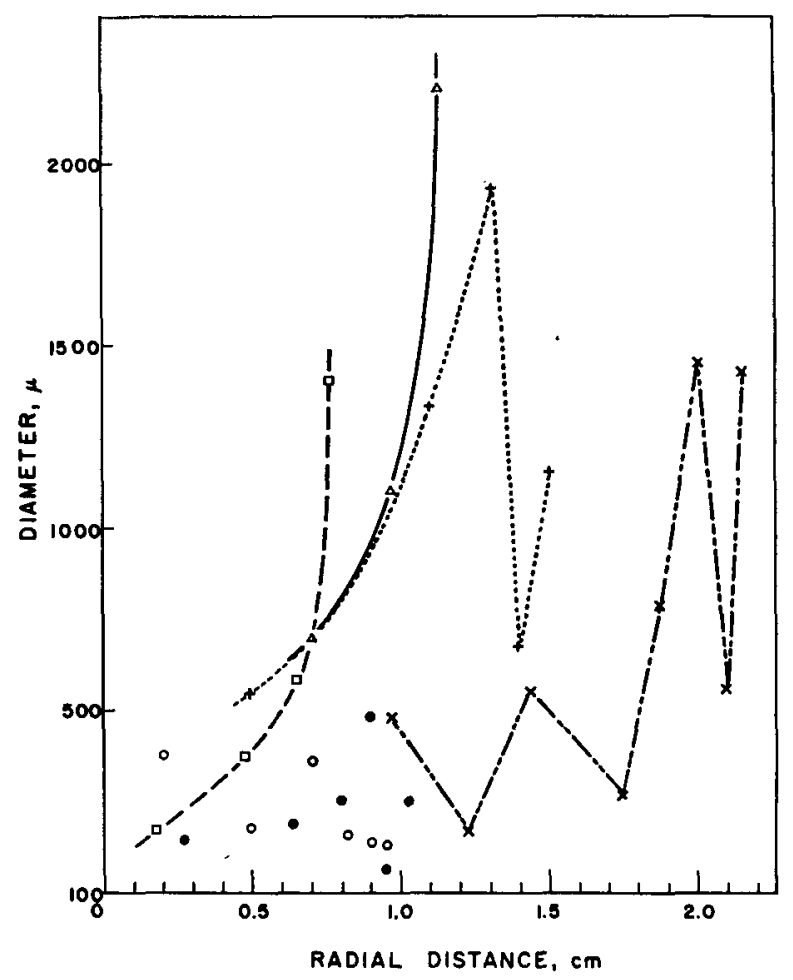

FIG. 6. The largest particle found in different hailstones vs. radial distance; open circles ( $\mathrm{B}-6.30 .65$ No. 1 ), filled circles ( $\mathrm{B}-$ 6.30.65 No. 2), triangles (CSU-7.8.64 No. 2), squares (CSU7.8.64 No. 3), crosses (CSU-6.21.64 No. 4), and X's (CSU6.21 .64 No. 5 ). 
TABLE 5. The largest particle found in different layers of a hailstone.

\begin{tabular}{|c|c|c|c|c|c|c|c|}
\hline Hailstorm & $\begin{array}{c}\text { Hailstone } \\
\text { number }\end{array}$ & $\begin{array}{c}\text { Radial } \\
\text { distance } \\
(\mathrm{cm})\end{array}$ & $\begin{array}{l}\text { Particle } \\
\text { dimensions } \\
(\mu)\end{array}$ & Hailstorm & $\begin{array}{l}\text { Hailstone } \\
\text { number }\end{array}$ & $\begin{array}{c}\text { Radial } \\
\text { distance } \\
(\mathrm{cm})\end{array}$ & $\begin{array}{c}\text { Particle } \\
\text { dimensions } \\
(\mu)\end{array}$ \\
\hline \multirow[t]{27}{*}{$B-6.30 .65$} & \multirow[t]{6}{*}{1} & $0.94-0.96$ & $320 \times 150$ & \multirow{10}{*}{$\begin{array}{c}\text { CSU-7.8.64 } \\
\text { con't. }\end{array}$} & 1 & $0.58-0.87$ & $120 \times 100$ \\
\hline & & $0.88-0.94$ & $380 \times 100$ & & con't. & & \\
\hline & & $0.76-0.88$ & $200 \times 160$ & & \multirow[t]{3}{*}{2} & $1.08-1.16$ & $2200 \times 2200$ \\
\hline & & $0.61-0.76$ & $420 \times 300$ & & & $0.87-1.08$ & $1100 \times 1100$ \\
\hline & & $0.40-0.61$ & $420 \times 150$ & & & $0.65-0.87$ & $800 \times 600$ \\
\hline & & $0-0.40$ & $020 \times 150$ & & \multirow[t]{5}{*}{3} & $0.70-0.82$ & $2000 \times 800$ \\
\hline & \multirow[t]{5}{*}{2} & $1.00-1.05$ & $500 \times 210$ & & & $0.58-0.70$ & $600 \times 580$ \\
\hline & & $0.95-1.00$ & $200 \times 140$ & & & $0.36-0.58$ & $450 \times 300$ \\
\hline & & $0.85-0.95$ & $580 \times 410$ & & & $0-0.36$ & $460 \times 70$ \\
\hline & & $0.76-0.85$ & $410 \times 300$ & & & & \\
\hline & & $\begin{array}{r}0.56-0.76 \\
0-0.56\end{array}$ & $\begin{array}{l}300 \times 280 \\
400 \times 80\end{array}$ & \multirow[t]{2}{*}{ CSU-6.21.64 } & 2 & $1.46-1.63$ & $1300 \times 1100$ \\
\hline & \multirow{4}{*}{3} & & & & \multirow[t]{4}{*}{3} & $1.31-1.43$ & $1120 \times 900$ \\
\hline & & $1.47-0.70$ & $2000 \times 200$ & & & $1.08-1.31$ & $1300 \times 600$ \\
\hline & & $0-0.47$ & $1600 \times 75$ & & & $0.84-1.08$ & $250 \times 280$ \\
\hline & & & & & & $0-0.84$ & $250 \times 160$ \\
\hline & \multirow[t]{2}{*}{4} & $\begin{array}{r}0.57-0.71 \\
0-0.57\end{array}$ & $\begin{array}{l}800 \times 700 \\
320 \times 150\end{array}$ & & \multirow[t]{4}{*}{4} & $1.47-1.53$ & $2000 \times 300$ \\
\hline & & & & & & $1.36-1.47$ & $720 \times 620$ \\
\hline & \multirow[t]{2}{*}{5} & $0.71-0.81$ & $3800 \times 300$ & & & $1.22-1.36$ & $2560 \times 1300 \times 90$ \\
\hline & & $0.54-0.71$ & $3400 \times 1400$ & & & $\begin{array}{r}1.02-1.22 \\
0-1.02\end{array}$ & $\begin{array}{c}1400 \times 1270 \\
690 \times 410\end{array}$ \\
\hline & \multirow[t]{3}{*}{6} & $0.85-0.96$ & $2500 \times 600$ & & \multirow{8}{*}{5} & & \\
\hline & & $0.73-0.85$ & $150 \times 100$ & & & $2.12-2.17$ & $1600 \times 1250$ \\
\hline & & $0.50-0.73$ & $2500 \times 70$ & & & $2.04-2.12$ & $820 \times 300$ \\
\hline & \multirow{5}{*}{7} & $096-102$ & $1500 \times 30$ & & & $\begin{array}{l}1.94-2.04 \\
181-1.94\end{array}$ & $\begin{array}{l}2000 \times 900 \\
1050 \times 530\end{array}$ \\
\hline & & $0.83-0.96$ & 1000 А- & & & $1.68-1.81$ & $400 \times 350$ \\
\hline & & $0.67-0.83$ & $900 \times 600$ & & & $1.53-1.68$ & $950 \times 60$ \\
\hline & & $0-0.67$ & $1400 \times 400$ & & & $1.35-1.53$ & $370 \times 290$ \\
\hline & & & & & & $1.12-1.35$ & $340 \times 210$ \\
\hline CSU-7.8.64 & 1 & $1.11-1.23$ & $2800 \times 700$ & & & $0.81-1.12$ & $670 \times 290$ \\
\hline
\end{tabular}

capture for the above analysis at this time. The point which should be emphasized is that the spatial distribution of insoluble solid particles within a hailstone may be a fingerprint which will permit deciphering the history of the hailstone.

The concentration of particles in any ice layer of a hailstone corresponds to the concentration present in cloud particles at the time the layer was formed, assuming evaporation was not taking place. A layer of lower concentration may indicate either growth from vapor at an altitude where fewer solid particles were available for capture or from cloud droplets relatively free of solid particles. A layer of higher concentration may indicate growth from cloud particles with higher concentrations of solid particles, e.g., water droplets which lost some water content due to evaporation or ice crystals which accumulate more solid particles during their growth from vapor in the presence of evaporating water droplets (Vittori, private communication). The number of fluctuations and their amplitude may also indicate the residence time at different altitudes, which in turn may permit drawing the complete trajectory of a given stone.

The study of different sizes of particles within layers indicates that larger particles are preferentially captured by a growing stone. This may indicate the higher efficiency of aerodynamic capture of particles which may not be associated with cloud droplets. These particles are of the same size range as cloud droplets in hail-producing clouds and, therefore, their larger values of constant $\gamma$ indicate not a higher rate of evaporation but rather capture to some extent of free aerosol particles. Very large particles (above $100 \mu$ diameter) are so few that the error in their estimation may lead to wrong conclusions though the $\gamma$ for them differ little from the $\gamma$ for the $45-100 \mu$ range. The large difference in number concentration of $5-13 \mu$ diameter size range permits using this size only for analysis. The addition of the remaining particles changes the second decimal place only. But the mode by which the other classes change may throw light on the concentration of aerosol particles within the air parcels where the particular hailstone was residing.

In Fig. 3, No. 2 and No. 4, the peripheral concentration of particles larger than $100 \mu$ diameter is increasing much more rapidly than that of the $45-100 \mu$ range, indicating a) the presence of this size $(d>100 \mu)$ of particles in the path of a hailstone and $b$ ) the influence of aerodynamic capture. In stone No. 5 (Fig. 3) the increase of $100 \mu$ and larger diameter particles is threefold for a peripheral radial growth of $0.05 \mathrm{~cm}$. The aerodynamic capture probably is predominant here, but the 
transfer of solid particles from other forms of precipitation upon collision should not be ruled out. The largest particles found in different layers of hailstones may also contribute to better understanding of their formation.

Hailstones No. 1 and No. 2 from a hailstorm which passed through Boulder, Colorado were collected four miles northeast of Boulder. Ice shells of these hailstones contained particles of average dimensions, $400 \times 200 \mu$. Hailstones collected in Boulder from the same storm contained particles of the approximate size $1700 \times 400 \mu$. The largest particle was $3.4 \times 1.4 \mathrm{~mm}$ (B-6.30.65 No. 5, Table 5).

Updrafts found in hailstorms can easily lift a particle as large as those found in the Boulder hailstorm. But during upward transport, larger particles will be removed by sedimentation, producing a particle size gradient. It could be concluded that hailstones collected four miles from Boulder originated in a storm cell at higher altitudes than those collected in Boulder. Another explanation may be that stronger updrafts were present when the storm originated in Boulder. Hailstones collected in northern Colorado always contained smaller particles inside the core, and particle size increased with radial distance (Fig. 6). If these very large solid particles are collected by a hailstone directly from air (that is, they are not first scavenged by raindrops and then transferred from them into a hailstone during the hailstone-raindrop collision), and if the particle size gradient versus altitude were present in such a turbulent atmosphere, then it can be concluded that these hailstones grew during their terminal fall through the clouds. The residence distances calculated for the grow th from $R_{0}$ to $R$ for two hailstones from different storms were 12 and $2 \mathrm{~km}$. Their diameters changed from 2.04 to $3.06 \mathrm{~cm}$ and from 1.34 to $2.04 \mathrm{~cm}$, respectively. Assuming that the growth from $R_{0}$ to $R$ is taking place during the downward fall of a hailstone, the time available for this growth was 14 and $3 \mathrm{~min}$, respectively, using average terminal velocity of 14 and $12 \mathrm{~m} \mathrm{sec}^{-1}$.

Rain water and snow crystals from hailstorms were not analyzed. Table 6 presents the average results of ten measurements of particle size and concentration performed on rain and snow collected at different times in 1965. Rain was collected in Boulder and snow was collected in Winter Park, Colorado, at different locations. The absence of $13-100 \mu$ diameter particles in snow collected in the forested area (at $2500 \mathrm{~m}$ elevation) and at an elevation of $3100 \mathrm{~m}$ (Twin Cones area) is again rather difficult to explain. Possibly, there is a particle size separation process taking place in clouds to such an extent that a large particle size range, in this case $13-100 \mu$, could be removed leaving conditions for
TABLE 6. Average concentration of particles in rain water and snow.

\begin{tabular}{lcccccc} 
& & \multicolumn{5}{c}{$\begin{array}{c}\text { Concentration }\left(\mathrm{cm}^{-3}\right) \text { for particle } \\
\text { diameter range }(\mu)\end{array}$} \\
Precipitation & Date & $5-13$ & $13-45$ & $45-100$ & $100-$ \\
\hline Rain & 8.18 .65 & (4) 1.53 & (3) 1.25 & (2) 1.16 & (1) 2.5 \\
Snow & & & & & \\
$\quad$ In the forest & 3.22 .65 & (2) 4.84 & 0 & 0 & (1) 7.0 \\
At the lodge & 3.17 .65 & (3) 1.80 & (2) 2.16 & 0 & (1) 9.4 \\
At 3800 m & 2.22 .65 & (4) 1.20 & 0 & 0 & (2) 1.1
\end{tabular}

The numbers in parentheses indicate the power of 10 by which tabulated values are to be multiplied.

formation of snow or cloud particles from which hail is formed. In this case, the cores of hailstones and snow will be free of this particle size class as found in several hailstones and snows (see Table 6) analyzed. Another explanation for a missing size class in snow could be as follows : fresh falling snow contained particles up to $13 \mu$ diameter where particles larger than $100 \mu$ were deposited later as ground contamination.

Absence of solid particles in some zones in ice can be explained by the interaction between particles and a moving ice-liquid interface. In this case, particles are pushed ahead into the liquid phase and are travelling along with the ice-water interface as it advances. This mechanism can produce practically particle-free ice in a hailstone but of a limited thickness because of the finite life time of a hailstone. The largest particles found in this "particle-free" ice will give information on the rate of advance of the ice front if it is smooth. This size will correspond to the critical size which cannot be displaced by a moving interface and will be trapped in the ice phase. What cannot be explained at this time is the fact that the intermediate class of particles is missing in some ice layers. At first it was thought that the instrument did not count these sizes of particles due to some mechanical or electrical malfunction. However, addition of $44-53 \mu$ diameter zinc sulfide particles to melted snow samples has shown that there was no instrumentation failure.

Additional information regarding formation of hailstones was obtained by slicing hailstones, sublimating ice from a slice, and subsequently estimating area density of particles of different size ranges. In this method, however, the diameter size range larger than $100 \mu$ is practically missing because the number of these particles is small and only a small fraction of them can be found in the microscopic viewing area. The results for particle size ranges $d<5 \mu, 5 \leq d<10 \mu, 10 \leq d<25 \mu$ and $d \geq 25 \mu$ found in a hailstone from the storm of 30 June 1965 in Boulder are reported for one slice in Figs. 7, 8, 9 and 10. 


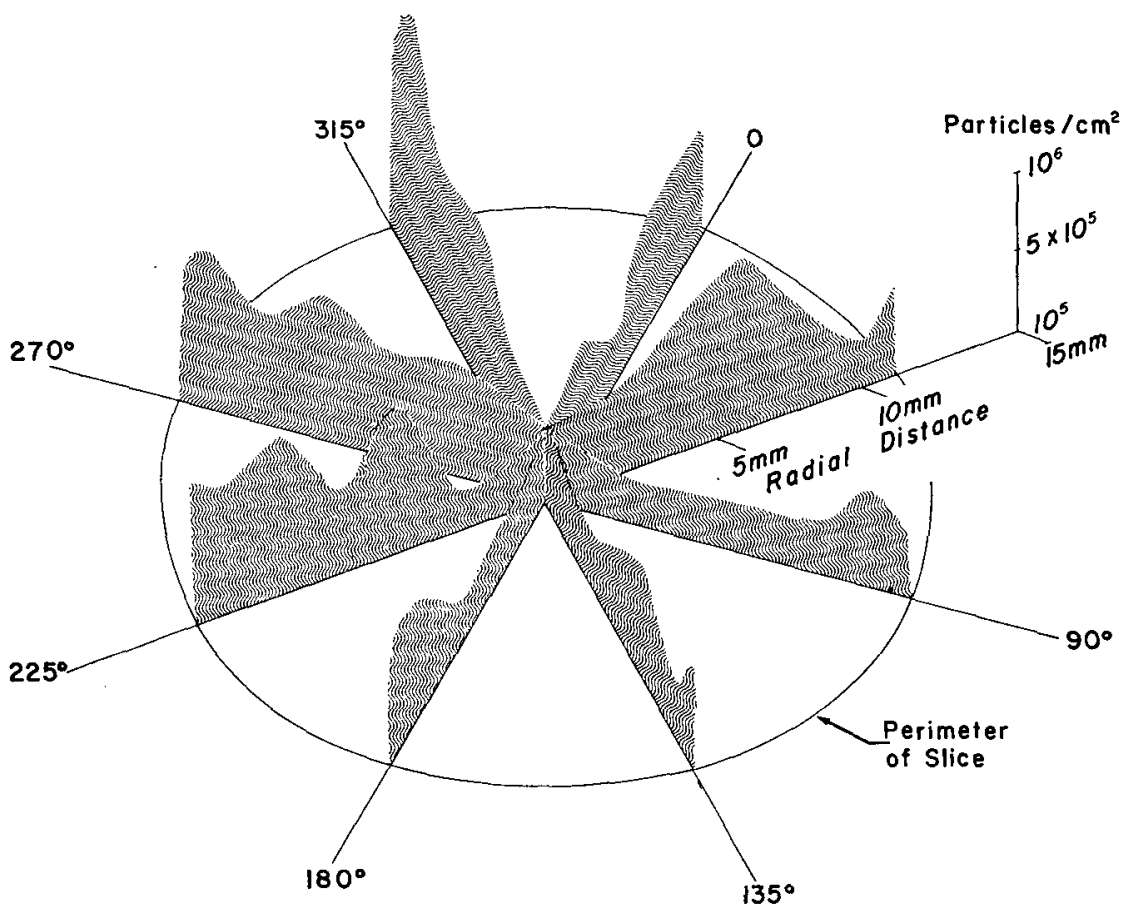

FIg. 7. Particle area density vs. radial distance for particles of $d<5 \mu$ in $1.5 \mathrm{~mm}$ slice of a hailstone.

The microscopic examination of the area distribution of particles of different sizes within the slice has shown that the values obtained using the Coulter counter for particle concentration vs. different radial distance are of the same order of magnitude. Spatial distribution of particles within shells can indicate the orientation of a hailstone during its fall. In this case, the hailstone was traveling with frontal area around $335^{\circ}$ (Fig. 7). The smallest accumulation of particles exists in the $160^{\circ}$ zone, indicating the downwind zone. Particles of all sizes were displaced from the $335^{\circ}$ zone probably by air pressure and movement of liquid water present on the surface into the $45-90^{\circ}$ and $225-270^{\circ}$ zones (Figs. 8 and 9).

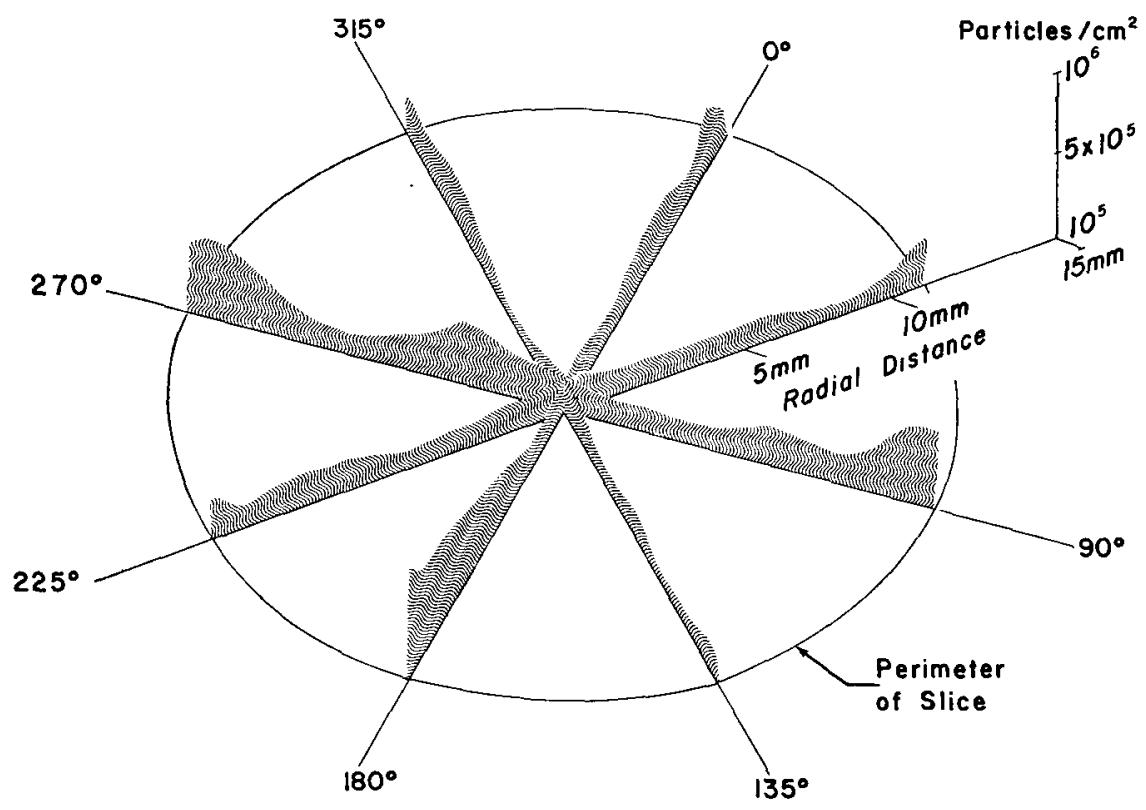

Frg. 8. Particle area density vs. radial distance for particles of $5 \leq d<10 \mu$ in $1.5 \mathrm{~mm}$ slice of a hailstone. 


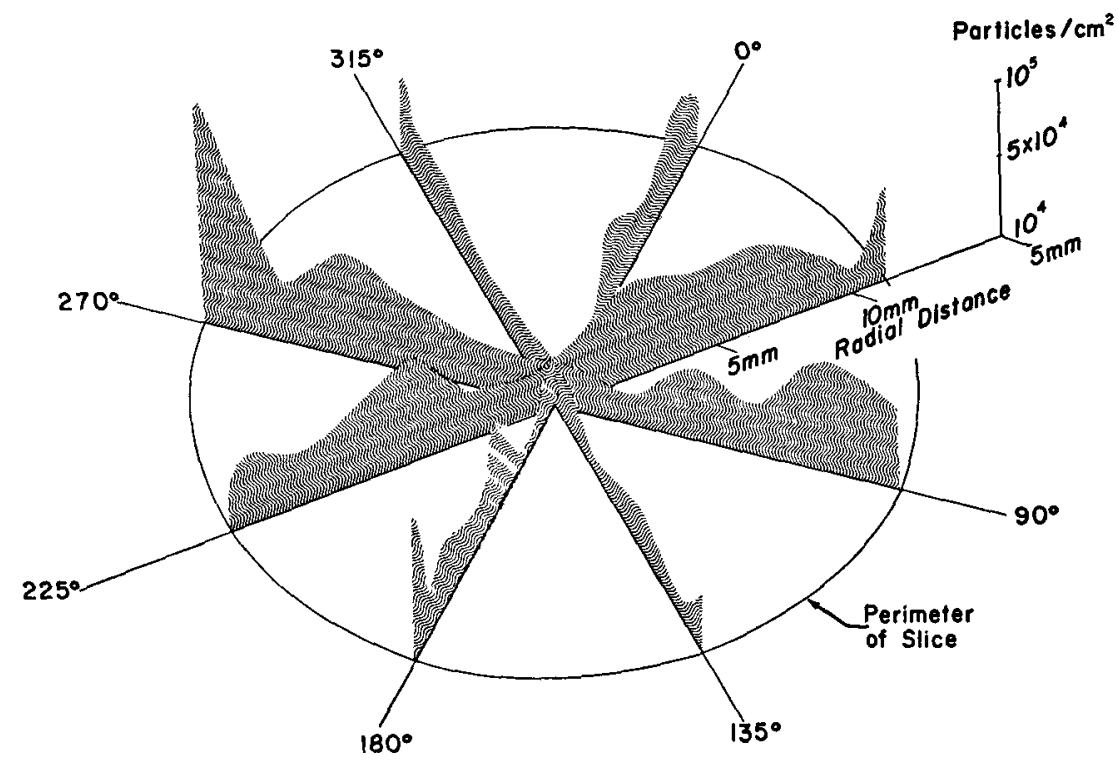

Fig. 9. Particle area density vs. radial distance for particles of $10 \leq d<25 \mu$ in $1.5 \mathrm{~mm}$ slice of a hailstone.

Further investigation has shown the existence of hailstones in which no pattern of spatial distribution of particles can be detected. Another category was also found in which solid particles are heavily concentrated around some points within a hailstone.

\section{Conclusions}

On the basis of the determination of the spatial distribution of solid water-insoluble particles in hailstones, one might hope in the future to contribute to solutions to the following problems:
1) Orientation of a hailstone during its residence time in the atmosphere;

2) Time for the growth of a hailstone under conditions of water evaporation or ice sublimation from its surface under the straight-line relationship between radial distance and particle concentration;

3) Altitude at which particular ice layers in a hailstone were formed or, knowing altitude from other measurements, the environmental conditions under which they form; and

4) A complete trajectory of a hailstone.

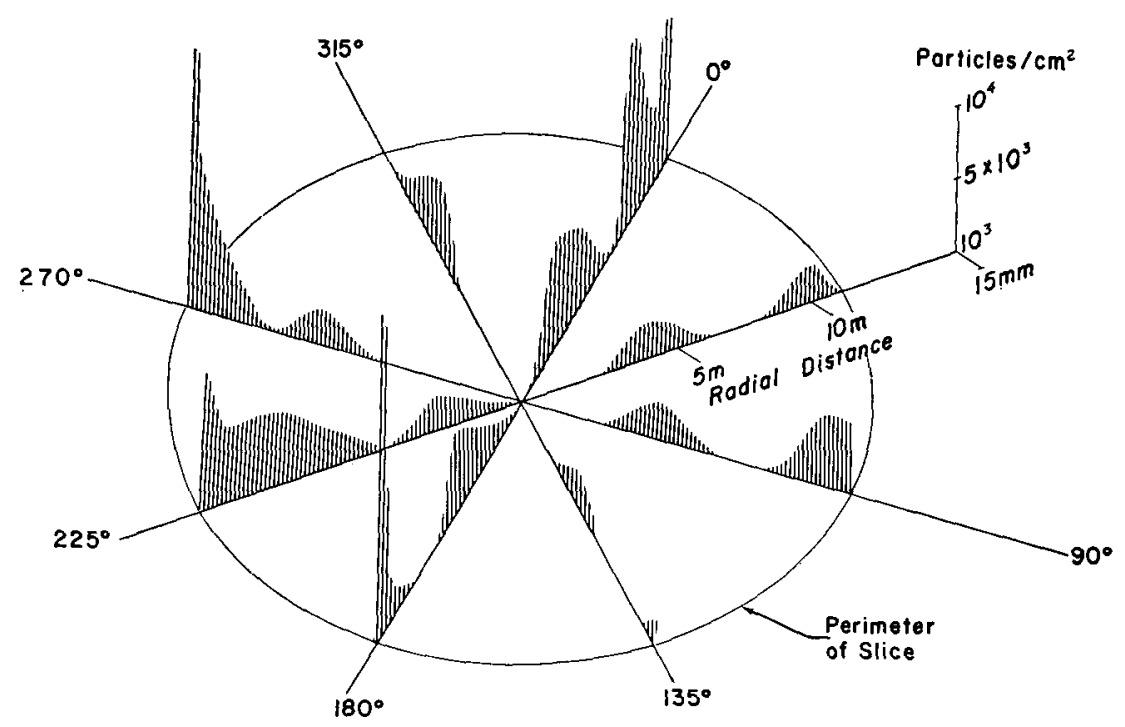

Fig. 10. Particle area density vs. radial distance for particles of $d \geq 25 \mu$ in $1.5 \mathrm{~mm}$ slice of a hailstone. 
At this time it was possible to deduce concentration of solid particles present in cloud droplets from the analysis of hailstones. To resolve the trajectory of a hailstone, concentration and size distribution of solid water-insoluble particles should be determined in cloud particles and within the storm cell in which it originated. In addition to this, hailstones practically without liquid water were examined. When liquid water content in a hailstone is large, solid particles can be redistributed with the advancing ice fronts upon freezing for storage. This type of hailstone may require a different technique of analysis. Interpretation of hailstone build-up from analysis of spatial distribution of solid particles within the hailstone will then permit thermodynamic calculations of hailstone formation and growth.

Acknowledgments. The author would like to acknowledge many hours of stimulating discussions with Profs. R. Braham, University of Chicago, O. Vittori, University of Bologna, Italy, and Drs. G. Goyer and C. Knight, National Center for Atmospheric Research. The author would also like to acknowledge the great patience and skill with which Miss S. Brannon, Carleton College, Northfield, Minn., performed the particle size analysis reported in this paper.

\section{REFERENCES}

Battan, L. J., 1965 : A view of cloud physics and weather modification in the Soviet Union. Bull. Amer. Meteor. Soc., 46, 309-316.

Gregory, P. H., 1961: The Microbiology of the Atmosphere. New York, Interscience Publishers, Inc., $251 \mathrm{pp}$.

Hoekstra, P., and R. D. Miller, 1965: Movement of water in a film between glass and ice. Cold Regions Research and Engineering Laboratory, Hanover, N. H., DA Task IV 014501B52A31, 8 pp.

List, R., 1961: Physical methods and instruments for characterizing hailstones. Bull. Amer. Meteor. Soc., 42, 452-466.

- - 1965: The mechanism of hailstone formation. Proc. International Conference on Cloud Physics, Tokyo, Japan, 481-491.

Uhlmann, D. R., B. Chalmers and K. A. Jackson, 1964: Interaction between particles and a solid-liquid interface. $J$. $A p p l$. Phys., 35, 2986-2993.

Vittori, O., and R. Pesaresi, 1964: Analisi microchimica della grandine. Ric. Sci., 34, serie 2, parte II-A, 5, 31-42. 